\title{
SNP array and FISH analysis of a proband with a 22q13.2- 22qter duplication shed light on the molecular origin of the rearrangement
}

\author{
Chiara Magri $^{{ }^{*}}$, Eleonora Marchina ${ }^{1}$, Valeria Bertini ${ }^{1}$, Michele Traversa $^{1}$, Giulia Savio ${ }^{1}$, Alba Pilotta ${ }^{2}$ \\ and Giovanna Piovani ${ }^{1}$
}

\begin{abstract}
Background: In about one third of healthy subjects, the microscopic analysis of chromosomes reveals heteromorphisms with no clinical implications: for example changes in size of the short arm of acrocentric chromosomes. In patients with a pathological phenotype, however, a large acrocentric short arm can mask a genomic imbalance and should be investigated in more detail. We report the first case of a chromosome 22 with a large acrocentric short arm masking a partial trisomy of the distal long arm, characterized by SNP array. We suggest a possible molecular mechanism underlying the rearrangement.

Case presentation: We report the case of a 15 -year-old dysmorphic girl with low grade psychomotor retardation characterized by a karyotype with a large acrocentric short arm of one chromosome 22.

Cytogenetic analysis revealed a normal karyotype with a very intense Q-fluorescent and large satellite on the chromosome 22 short arm. Fluorescence in situ hybridisation analysis showed a de novo partial trisomy of the 22q13.2-qter chromosome region attached to the short arm of chromosome 22. SNP-array analysis showed that the duplication was $8.5 \mathrm{Mb}$ long and originated from the paternal chromosome. Haplotype analysis revealed that the two paternal copies of the distal part of chromosome 22 have the same haplotype and, therefore, both originated from the same paternal chromosome 22. A possible molecular mechanism that could explain this scenario is a break-induced replication (BIR) which is involved in non-reciprocal translocation events.

Conclusion: The combined use of FISH and SNP arrays was crucial for a better understanding of the molecular mechanism underlying this rearrangement. This strategy could be applied for a better understanding of the molecular mechanisms underlying cryptic chromosomal rearrangements.
\end{abstract}

Keywords: SNP-array, CNV, Translocation, Duplication, 22q13.2, 22qter, BIR

\section{Background}

In about one third of healthy subjects, the cytogenetic analysis of chromosomes reveals heteromorphisms with no clinical implications: for example changes in size of the short arm of acrocentric chromosomes These regions include three cytogenetic bands in which there are several types of tandem repeated DNA sequences: satellites I, II, III, IV, distal beta-satellite, multiple copies of rRNA genes, proximal beta satellite DNA, and telomere sequences [1-3].

\footnotetext{
* Correspondence: chiara.magri@unibs.it

${ }^{1}$ Biology and Genetics Division, Department of Molecular and Translational Medicine, University of Brescia, Viale Europa 11, 25123 Brescia, Italy Full list of author information is available at the end of the article
}

A change in size of the short arm of an acrocentric chromosome is considered as a benign variant, however, the detection of a variant chromosome, in a subject with an abnormal phenotype, should be investigated in more detail using cytogenetic and molecular technologies. Ravnan et al., [4] found a group of unbalanced translocations, involving a sub-telomere region of an acrocentric chromosome, and showed that apparent acrocentric short arm polymorphisms can indeed represent significant genomic imbalances.

In this report, we describe the combined use of fluorescence in situ hybridisation (FISH) and single nucleotide polymorphism (SNP) array technologies to study the case of a large acrocentric short arm of a chromosome 22 


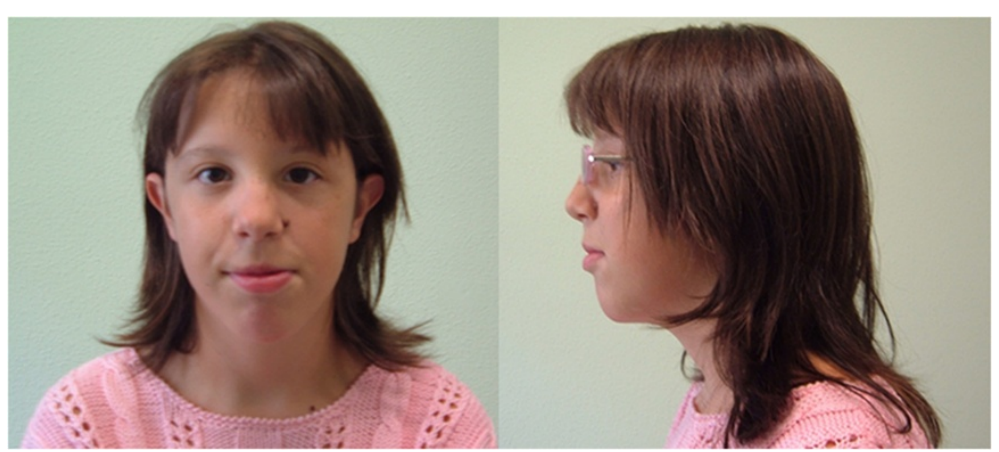

Fig. 1 Photographs of the patient at the age of 15. Frontal and lateral view of the proband showing mild dysmorphic traits

which masks a de novo trisomy of a distal portion of the long arm of chromosome 22 in a 15-year-old dysmorphic girl with mild psychomotor retardation. Only 19 cases of pure partial trisomy involving the distal long arm of chromosome 22 have been reported [5-19] three of which have been characterized using a CGH-array [13, 14, 19]. We believe that the case reported in this paper is the first analysed with SNP array. In most of the pure trisomy of distal 22q, duplication is the consequence of a de novo non-reciprocal translocation on the short arm of an acrocentric chromosome or a recombination event of a pericentric inversion in the parent. However, the molecular mechanisms underlying this chromosome rearrangement remain unclear [9].

We demonstrate how the combined use of FISH and SNP genotype array information may be useful to determine the parental origin of the duplicated portion of chromosome 22 and to hypothesize a possible molecular mechanism of formation.

\section{Case presentation}

\section{Clinical Report}

The proband was the second of two sisters born from healthy, non-consanguineous, Caucasian parents. At the time of birth, the mother was 32 and the father was 28 years old; the sister is healthy and familiar history was negative for malformation or developmental delay. The patient was born at 42 weeks by caesarean section because of fetal bradycardia; maternal gestational diabetes was evident from the fifth month of pregnancy, no prenatal diagnosis was made. The birth weight was $3.2 \mathrm{~kg}(25-50$ percentile), length was $49 \mathrm{~cm}$ (25 percentile), and the Apgar score was 8/10. The neonatal period was unremarkable, congenital hip dysplasia was immediately evident and specific support was applied for three months.

At five years of age, when starting school, a delay in psychomotor skills and language was present, cognitive performance was evaluated by the Wechsler Intelligence Scale for Children (WISCH), together with writing and reading tests. Low-grade mental retardation was evidenced. Psycho- pedagogical help was provided and the primary school was successfully completed. The growth was normal in terms of height, 25 percentile (Tanner scale), and weight however, until 11 years old, the bone age was delayed by two years compared to the chronological age, from 12 years, both ages were similar. Puberal development was at 12.5 years and menstrual cycles were regular.

At the last examination, the 14.9 year-old patient attended a secondary technical school, her height was $157.8 \mathrm{~cm}$ ( 25 percentile), weight was $44.2 \mathrm{~kg}$ (3 percentile), the head circumference was $50.5 \mathrm{~cm}(<3$ percentile) and sexual development had been completed.

Physical examination showed some dysmorphic features: microcephaly, long face, sparse eyebrows, telecanthus, low set and laterally protruding ears, long philtrum, macrostomia, prominent lower lip, large ear, gibbus deformity, bilateral clinodactyly of the fifth finger of hand (Fig. 1). No psychiatric signs were observed. Ultrasound examination of heart and other internal organs gave normal results.

\section{Materials and methods}

Chromosome preparations were obtained with phytohaemoagglutinin stimulated lymphocytes from a peripheral blood sample. Cultures were set up and harvested by standard methods. Cytogenetic analysis was performed using $\mathrm{Q}$ and G-banding at 450 bands resolution and using C, Nucleolus Organiser Region (NOR) and Distamycin A and 4',6diamidino-2-phenylindole (DA-DAPI) stainings according to the International System for Human Cytogenetic Nomenclature [20, 21]. The Chromoprobe Multiprobe-T System (Cytocell Ltd) was used to screen the subtelomeric regions according to the manufacturer's protocol.

FISH analysis was carried out by standard procedures as described previously [22]. Dual-colour FISH experiments were performed with Bacterial Artificial Chromosome (BAC) clones reported in Table 1 and provided by Chori (BAC PAC Resources Center Children's Hospital Oakland Research Institute in Oakland, California, USA). 4',6-Diamidino-2-Phenylindole, Dilactate (DAPI) staining was used for chromosome identification during FISH analysis. 
Table 1 BAC clones used for dual colour FISH experiments

\begin{tabular}{lllll}
\hline BAC clones & $\begin{array}{l}\text { Position on } \\
\text { Chr22 (Hg19) }\end{array}$ & Dual colour FISH & Notes \\
\cline { 3 - 4 } & 1st FISH & 2nd FISH & \\
\hline RP11-241G19 & $42,605,118-42,782,007$ & green & & Located outside the duplicated region \\
RP11-140115 & $46,097,912-46,268,798$ & & green & \\
RP11-164E23 & $50,841,120-50,995,394$ & red & red & The most telomeric RP11 probe available on Chr22q. \\
\hline
\end{tabular}

FISH signals were analysed using a Zeiss Axiophot microscope with a triple bandpass filter to simultaneously detect signals. Images were collected and merged using a cooled CCD camera.

The DNA of the proband and of her healthy parents were analysed with GeneChip Human Mapping $250 \mathrm{~K}$ $N s p$ I arrays (Affymetrix). The DNA was processed following instructions provided in the Affymetrix GeneChip Human Mapping $500 \mathrm{~K}$ Assay Manual. Initial analysis and quality assessment of the array data were performed using GTYPE 4.1 (Affymetrix). Copy number state determination was performed using CNAT4 software (Affymetrix) by comparing our samples with a reference set of 48 Hapmap samples available on the Affymetrix web
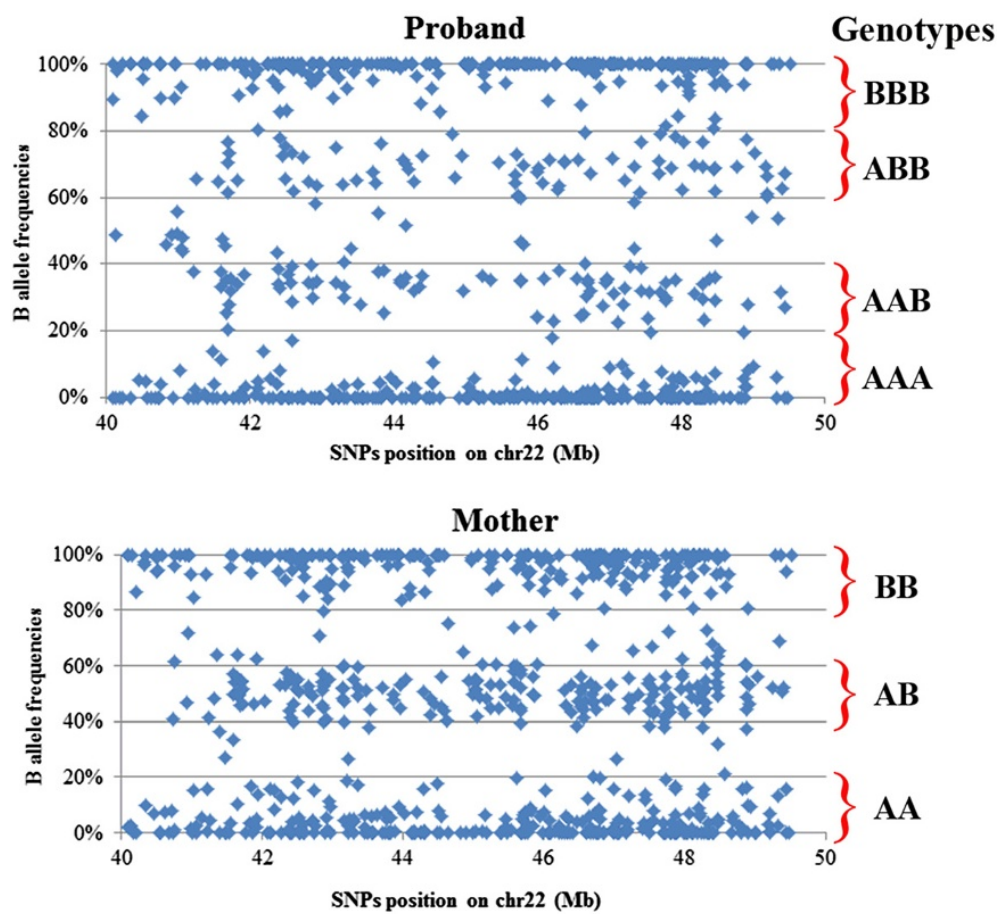

BB

AB

\section{AA}

Father

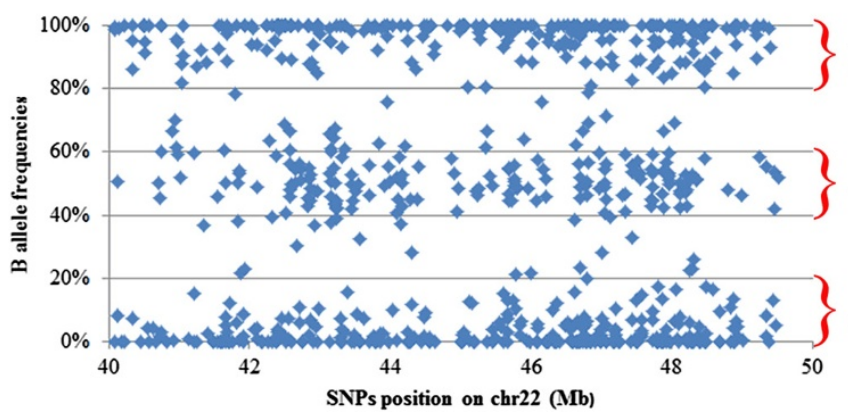

BB

$\mathbf{A B}$

Fig. 2 Scatterplots of the relative B allele intensity signals. On the right, B allele intensity signals for the SNPs in the duplicated portion of chromosome 22 in the proband and her parents, respectively. On the left, the genotype assigned to each probe according to their B allele frequencies 
A

The abnormal chr.22

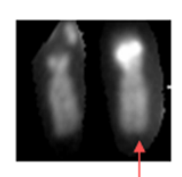

B

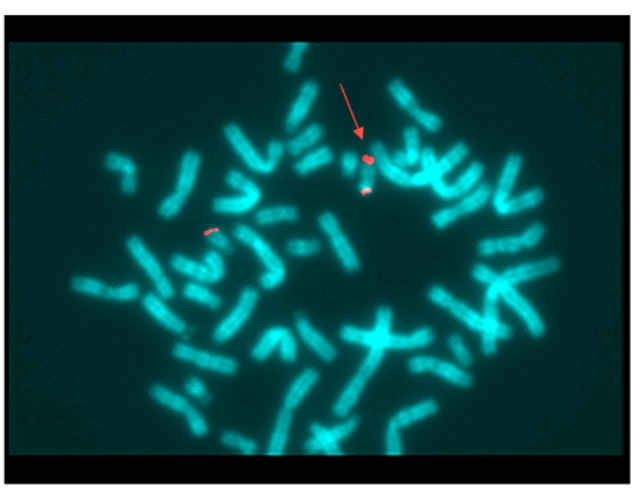

C

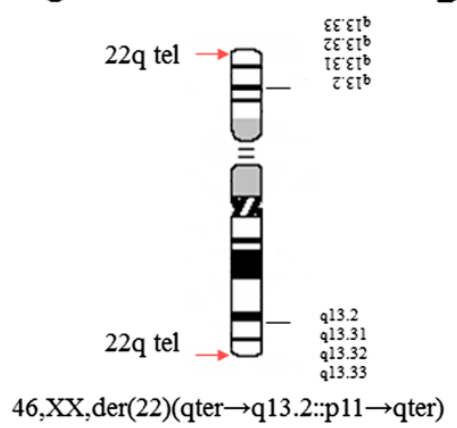

D

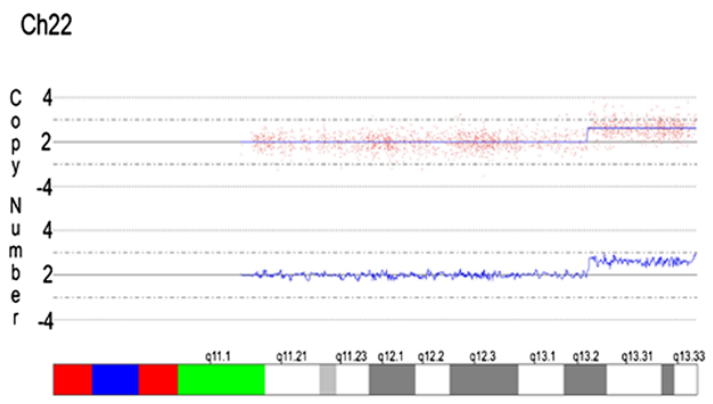

Fig. 3 Cytogenetic and molecular analyses. a chromosome 22 QFQ banded karyotype of the patient. The red arrow points to the abnormal chromosome 22. b FISH analysis with probes for the subtelomeric region of chromosome 22q (in red). An additional signal located on the short arm of chromosome 22. c The derivative ideogram of the chromosome 22q13.2-qter duplication; $\mathbf{d}$ Array analysis. Copy number state of chromosome 22 probes inferred by CNAT and reported as number of copies

site (http://www.affymetrix.com/support/technical/sample_ data/500k_data.affx).

Concerning the duplicated region, Affymetrix Power Tool software was used to extract "allele specific" signal values from the raw CEL file for those SNPs falling in the duplicated region. "Allele specific" refers to the fact that for each SNP, there is a signal measure for the A allele and a separate signal measure for the B allele. The relative intensity signal of the $B$ allele was then used to assign the genotype. The homozygous $\mathrm{BB}$ genotype was assigned to $\mathrm{B}$ intensity values between 1 and $0.8, \mathrm{ABB}$ to values between 0.8-0.6, $\mathrm{AB}$ to values between $0.6-0.4$, $\mathrm{AAB}$ to values between $0.4-0.2$ and $\mathrm{AA}$ to values between 0.2-0.0 (Fig. 2).
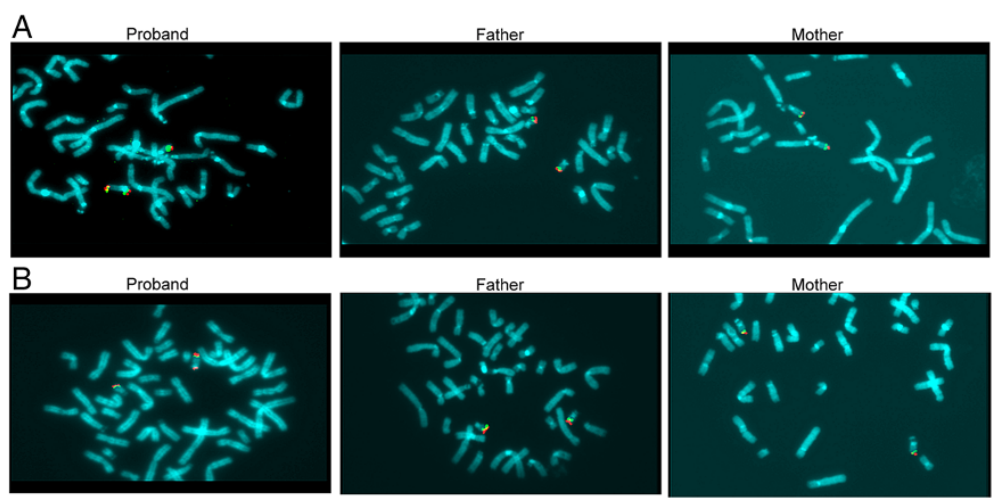

Fig. 4 Dual Colour FISH analyses. a FISH analysis with probes RP11-140115 (green) and RP11-164E23 (red). In the proband, two additional hybridisation signals on the abnormal short arm of chromosome 22 are visible, whereas in the parents only two signals in the correct orientation are visible on the long arm of chr22. b FISH analysis with probes RP11-241G19 (green) and RP11-164E23 (red). The RP11-241G19 probe is located outside the duplicated region and displays only one hybridisation signal on the long arm of chr22 in all the subjects 
Table 2 Allele transmission pattern for proband heterozygous SNPs in the 22q duplicated region

\begin{tabular}{lllll}
\hline $\begin{array}{l}\text { Proband } \\
\text { heterozygous } \\
\text { genotypes }\end{array}$ & $\begin{array}{l}\text { Mother } \\
\text { genotype }\end{array}$ & $\begin{array}{l}\text { Father } \\
\text { genotype }\end{array}$ & $\begin{array}{l}\text { Parent transmitting } \\
\text { two alleles (alleles) }\end{array}$ & $\begin{array}{l}\text { Tot. } \\
\text { SNPs }\end{array}$ \\
\hline AAB & AB & AA & Mat(AB) or Pat(AA) & 32 \\
& AB & AB & Not informative & 5 \\
& BB & AA & Pat(AA) & 13 \\
& BB & AB & Pat(AA) & 21 \\
ABB & AA & AB & Pat(BB) & 17 \\
& AA & BB & Pat(BB) & 22 \\
& AB & AB & Not informative & 6 \\
& AB & BB & Mat(AB) or Pat(BB) & 20 \\
Total SNPs & BB & AB & Mat(BB) or Pat(AB) & 1 \\
\hline
\end{tabular}

The bioinformatics analysis of the sequences involved in the chromosomal rearrangement was carried out using the University of California Santa Cruz (UCSC) Genome Browser (http://genome.ucsc.edu/) on the GrCh37 Feb 2009 release of the human genome.

\section{Results}

Cytogenetic analysis of the proband revealed a normal karyotype, however Q banded analysis showed the presence of one normal chromosome 22 and one submetacentric chromosome 22 with a very intense Q-fluorescent and large satellite on the short arm (Fig. 3a). C banding confirmed the presence of only one centromere, staining with silver nitrate revealed the presence of a NOR in the $\mathrm{p}$ arm, while DA-DAPI staining was negative.

FISH with telomere set probes revealed an additional positive hybridisation signal of the specific subtelomere probe of the 22 chromosome long arm on the large satellite of the short arm of chromosome 22 (Fig. 3b). To rule out the presence of cryptic imbalances in the genome and to confirm our results, we performed further investigations using BAC clones (RP-11) specific for different regions of chromosome $22 \mathrm{q} 13.2$ to characterize the duplicated region. Dual-colour FISH with BAC clones RP11-140I15 and RP11-164E23 showed two hybridisation signals on the abnormal short arm of chromosome 22, whereas dual-colour FISH with BAC clones RP11-241G19 and RP11-164E23 revealed only one hybridisation signal of BAC RP11-164E23 on the abnormal short arm of chromosome 22. Based on these findings, we determined the karyotype to be: 46,XX, ish $\operatorname{der}(22)$ (qter $\rightarrow$ q13.2::p11 $\rightarrow$ qter) $\quad$ [21], (Fig. 3c). FISH with telomeric and with the same BAC clones revealed normal results in the parents: no rearranged or inverted cryptic duplicated regions were highlighted (Fig. 4).
The extension of the duplication was characterized by SNP array. The analysis with Affymetrix GeneChip Human Mapping $250 \mathrm{~K}$ array showed that the duplication was $8.5 \mathrm{Mb}$ long and that the starting point mapped between 42806124 bp (SNP_A-1904331, first non-duplicated probe) and 42875479 bp (SNP_A-4229298 first duplicated probe) (Fig. 3d). The SNP array analysis of the parents' DNA confirmed the de novo origin of the duplication. To verify the paternal or maternal origin of the duplication, the Mendelian segregation of 137 probes, for which the proband was heterozygote $(\mathrm{AAB}$ or $\mathrm{ABB})$, were analysed (Table 2). Eleven probes were not informative since both parents were heterozygotes. For 53 SNPs (39\%), the duplicated allele could have been inherited from either the mother or the father. None of the duplicated alleles were clearly inherited from the mother, whereas for 73 SNPs (53\%), the duplicated allele was undoubtedly of paternal origin (Table 2). These data demonstrate that the duplicated portion of chromosome 22 arose on the paternal homologous.

Interestingly, the father transmitted always two identical alleles (AA or BB) to his daughter (Table 2). Only one probe circumvented this rule, likely related to a genotyping error in one of three samples. This demonstrates that the two paternal copies of the distal part of chromosome 22 have the same haplotype and, therefore, both originated from the same paternal chromosome 22 .

\section{Discussion}

Pure duplication of the distal long arm of chromosome 22 (22qter) seems to be extremely rare, in fact only 19 cases of pure trisomy 22qter have been described to date [5-19]. Fenstra et al., [9] subdivided pure trisomies 22qter into four groups according to the size of the duplicated region: large (22q12-qter), intermediate (22q13.1-qter), small (22q13.2qter) and smallest (22q13.3-qter) duplications. Usually the severity of the clinical phenotype correlates with the dimension of the duplicated region. However, with the exception of patients with the smallest duplications, who have a consistent clinical presentation leading to moderate/mild mental retardation, microcephaly and similar mild dysmorphic traits [14], patients with apparently the same duplicated region show a wide spectrum of phenotype variations. These differences could be due in part to the different genetic backgrounds in which the duplications arose, and in part to the often only approximate evaluation of the extent of the duplications. In fact, the only two patients with a molecular characterization of the breakpoint and analysed with an aCGH methodology were those reported by Peeters et al., [14] and Failla et al., [19].

We report a new case of a girl with a $8.5 \mathrm{Mb}$ duplication of the 22q13.2-qter region translocated to $22 \mathrm{p} 11$. This region includes 120 genes, 54 of which expressed (normalized expression $>1$ ) in the brain (according to EBI 
Table 3 Overview of clinical features of patients with a 22q13 to qter duplication

\begin{tabular}{|c|c|c|c|c|c|c|c|c|c|c|}
\hline & Petek et al. & $\begin{array}{l}\text { Biesecker et } \\
\text { al. }\end{array}$ & Schinzel & $\begin{array}{l}\text { Feenstra et } \\
\text { al. } \\
\text { (patient 1) }\end{array}$ & Our Patient & $\begin{array}{l}\text { Feenstra et al. } \\
\text { (patient 2) }\end{array}$ & $\begin{array}{l}\text { Feenstra et } \\
\text { al. } \\
\text { (patient 3) }\end{array}$ & $\begin{array}{l}\text { Okamoto et } \\
\text { al. } \\
\text { (patient 1) }\end{array}$ & Peeters et al. & Failla et al. \\
\hline $\begin{array}{l}\text { Duplicated } \\
\text { region }\end{array}$ & q13-qter & q13.2-qter & q13.2-qter & q13.2-qter & q13.2-qter & q13.3-qter & q13.3-qter & q13.3-qter & q13.3-qter & q13.3-qter \\
\hline $\begin{array}{l}\text { Duplication } \\
\text { cause }\end{array}$ & $\begin{array}{l}\text { der(19)t(19p; } \\
22 q) d n\end{array}$ & & $\operatorname{der}(6) t(6 ; 22) m a t$ & $\begin{array}{l}\operatorname{der}(21) t \\
(21 p ; 22 q)\end{array}$ & $\begin{array}{l}\operatorname{der}(22) t \\
(22 p ; 22 q) d n\end{array}$ & $\operatorname{der}(21) t(21 p ; 22 q)$ pat & $\begin{array}{l}\text { der(21)t } \\
(21 p ; 22 q) d n\end{array}$ & $\begin{array}{l}\operatorname{der}(22) t \\
(22 p ; 22 q) d n\end{array}$ & $\begin{array}{l}\text { der(22)t } \\
(22 p ; 22 q) d n\end{array}$ & \\
\hline Sex & M & $\mathrm{F}$ & $\mathrm{F}$ & $\mathrm{F}$ & $\mathrm{F}$ & M & M & $\mathrm{F}$ & $\mathrm{F}$ & $\mathrm{F}$ \\
\hline $\begin{array}{l}\text { Psychomotor } \\
\text { retardation }\end{array}$ & + & + & + & + & mild & moderate & mild & moderate & mild & $\begin{array}{l}\text { Moderate } \\
\text { Disorganized } \\
\text { schizophrenia }\end{array}$ \\
\hline Stature (height) & -2.5 S.D. & O S.D. & Short stature & -4 S.D. & O S.D. & -3 S.D & -3 S.D & $-3.2 \mathrm{SD}$ & $0 S D$ & \\
\hline Hypotonia & & + & & + & - & - & - & + & - & + \\
\hline \multicolumn{11}{|l|}{ Head } \\
\hline Microcephaly & + & + & + & + & + & + & + & + & + & + \\
\hline Brain abnormalities & & + & & - & - & - & - & - & - & - \\
\hline Sparse, fine hair & + & & & + & - & + & + & & & \\
\hline Prominent forehead & & & & + & - & + & + & + & + & \\
\hline Hypertelorism & - & + & + & + & + & - & + & + & - & + \\
\hline Palpebral fissures slant up & & & + & + & & - & - & - & & \\
\hline Narrow palpebral fissures & & & & + & & + & - & + & - & \\
\hline Epicanthic folds & + & & & + & + & - & - & & & \\
\hline Wide nasal bridge & & & & + & + & - & - & + & + & \\
\hline Low set ears & + & - & + & + & + & - & - & + & - & \\
\hline Dysplastic ears & & - & & - & - & - & - & + & - & \\
\hline Hearing loss & & + & - & - & - & - & - & - & - & \\
\hline Long philtrum & & & & - & + & - & - & & & \\
\hline Prominent upper lip & & & & + & - & + & + & & - & \\
\hline Prominent lower lip & & & & - & + & - & - & & - & \\
\hline Cleft lip & & + & - & - & - & & & & & \\
\hline Cleft palate & & + & + & + & - & - & - & - & - & \\
\hline \multicolumn{11}{|l|}{$\begin{array}{l}\text { Thorax, abdomen and } \\
\text { genitalia }\end{array}$} \\
\hline Renal malformations & + & & & + & - & - & - & - & - & \\
\hline Genital malformation & + & & & - & - & - & - & - & - & \\
\hline
\end{tabular}


Table 3 Overview of clinical features of patients with a 22q13 to qter duplication (Continued)

Dysplastic hip

Slender fingers

Clinodactyly fifth finger

Club foot, varus

Survival

Alive,

Alive, 9 years Alive, 8.5 years Alive, 5 years Alive,

Alive, 2 years

Alive,

40 years

Alive, 4 years Alive,

6.8 years

Alive, 20 years 
Expression Atlas database [23]) and 9 almost exclusively expressed in brain. Due to the high number of genes, the genotype phenotype correlation is, therefore, extremely difficult. Inside this region maps the gene SHANK3. Recently, it has been reported that SHANK3 dosage is critical for normal brain function and that its overexpression causes a hyperkinetic neuropsychiatric disorder characterized by manic-like behaviour and seizures consistent with synaptic excitatory/inhibitory imbalance [24]. Some of these characteristics have been observed, indeed, also in patients with the smallest 22q13.3-qter duplication $[9,19]$. Although our patient displays a mild phenotype, comparable to that of patients with the smallest 22q13.3-qter duplication (Table 3), she does not present psychiatric signs. She presents slight mental retardation, microcephaly and minor dysmorphic traits such as telecanthus, large low set and laterally protruding ears. However, she does not display major malformation like cleft palate, genital or kidney malformations as those observed in samples with the 22q13.2-qter duplication [7, 9, 15, 17] (Table 3).

It is generally assumed that 22qter duplications originate from the missegregation of a familial balanced translocation or through an abnormal recombination of a parental chromosomal inversion [25, 26]. Pure duplication of distal 22q, however, is usually the consequence of a de novo non-reciprocal translocation of the distal part of chromosome 22 on the short arm of an acrocentric chromosome $[9,13,14]$. In this sense, the case reported here is not an exception, since the duplication observed is due to a de novo unbalanced translocation of the 22q13.2-qter region on the short arm of the paternal acrocentric chromosome 22.

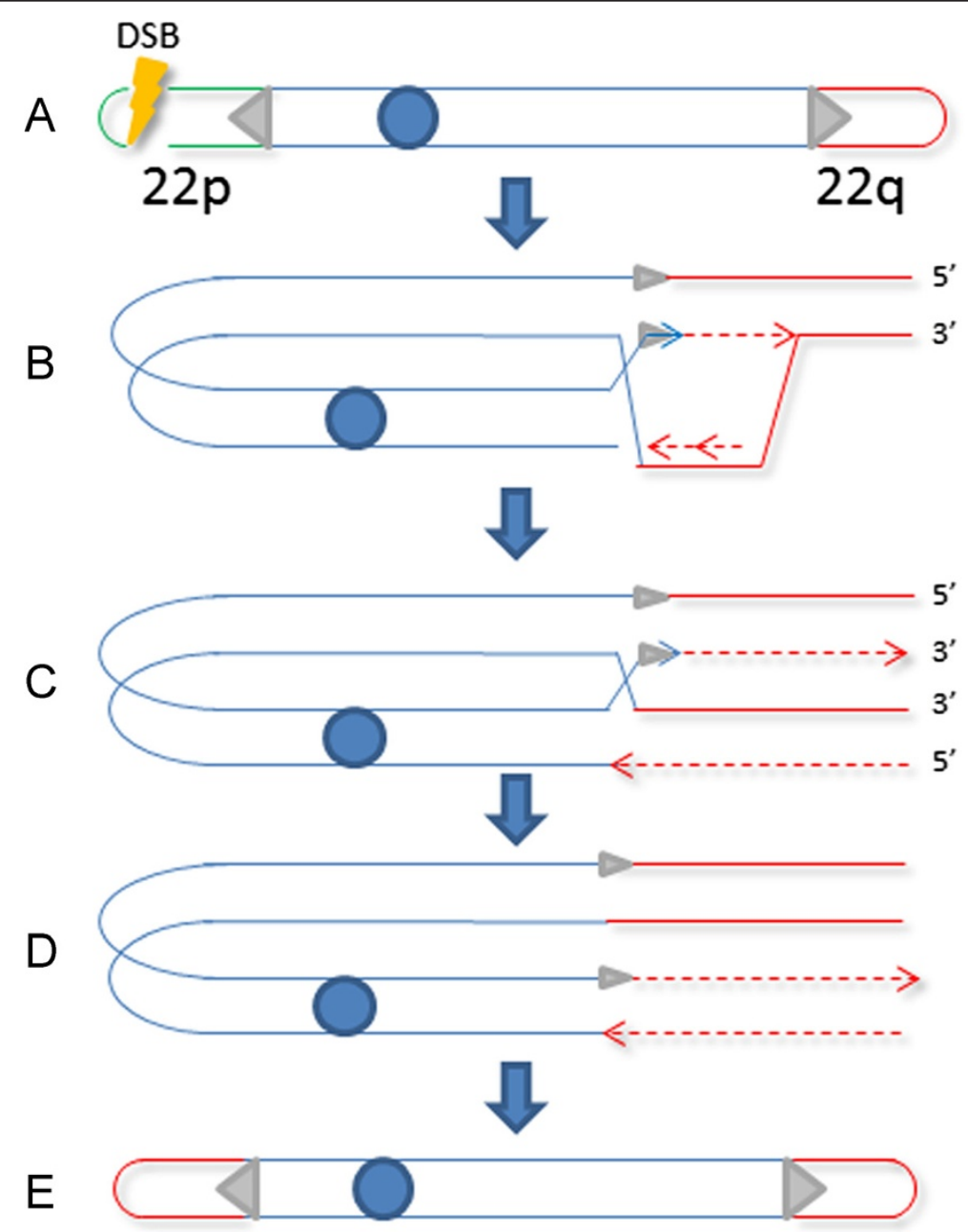

Fig. 5 Mechanism proposed to explain the formation of a non-reciprocal translocation of the duplicated 22q13.2-qter region. The grey triangles are short regions of microhomology. In green and red are the distal portions of the $22 p$ and $22 q$ arms, respectively. The picture is not to scale. a A DSB eliminates the telomere of the short arm. $\mathbf{b}$ The $3^{\prime}$ end proximal to the DSB invades a homolog sequence on the $q$ arm and induces the formation of a mono-directional replication fork. The arrow indicates the direction of the replication fork. $\mathbf{c}-\mathbf{d}$ The replication proceeds until the chromosome end is reached. e The distal portion of 22q (red) is duplicated and transposed inverted on 22p. See the discussion for details 
In order to clarify the mechanism underlying the $22 \mathrm{q}$ rearrangement in our patient, we analysed the genotype of the SNPs in the 22q13.2-qter region both in the proband and her parents. Genotype analysis showed that the duplication involved the paternal chromosome 22 and that the two paternal copies of the 22q13.2-qter region had an identical haplotype. This demonstrates that both copies originated from the same paternal chromosome. Interestingly, also in the patient reported by Failla et al., [19] the duplication involved the paternal chromosome: the microsatellite analysis revealed the presence of one maternal and two paternal alleles. Since genotype information is not available for the other cases of 22qter pure trisomies, it is not possible to conclude whether this is a general mechanism or if it represents only one possible molecular event. To clarify this it would therefore be desirable to also characterise the oldest cases using SNP array.

The fact that, in our patient, the two paternal 22q13.2qter copies have the same haplotype, enables us to rule out that the rearrangement is the result of a reciprocal translocation between the two paternal chromosomes 22 due to a non-allelic recombination during spermatogenesis.

Reciprocal translocations are the most common type of translocation and generally result from the swapping of chromosomal arms between heterologous chromosomes as a result of double strand breaks (DSBs) followed by nonallelic DNA repair mechanisms: non-homologous end join (NHEJ) or non-allelic homologous recombination (NAHR). Neither mechanism, however, is sufficiently adequate to describe the formation of non-reciprocal translocations, consequently other mechanisms need to be considered.

One model that could explain both the occurrence of a distal 22q intrachromosomal duplication and its nonreciprocal translocation on the short arm of an acrocentric chromosome, is a break-induced replication (BIR) which is involved in non-reciprocal translocation events in yeast [27-29]. BIR is a sub-pathway of the homologous recombination pathway used when only one DSB end shares homology with a template elsewhere in the genome (for example, in the case of telomere erosion).

In BIR, recombination is used to establish a unidirectional replication fork which can copy the template DNA till the end of the chromosome. If homologous sequences are located ectopically, BIR should result in the formation of a non-reciprocal translocation with loss of the telomeric part of the broken chromosome. It has been proposed that also regions of micro-homology are sufficient to induce a particular form of BIR defined micro-homologous-mediated BIR (MMBIR) [30]. Our hypothesis is that, on the distal part of the short arm of one chromosome 22, a DSB arose which resulted in the loss of the telomere. To stabilize the chromosome end, the 3 'end of the $22 \mathrm{p}$ probably invaded a micro- homologous sequence located on the other chromosome arm, thus forming a unidirectional replication fork. The replication from this site presumably then produced a non-reciprocal translocation in which the 22q13.2-qter part was duplicated distal to the chromosome end produced by the DSB. Figure 5 shows the model proposed.

Clearly a prerequisite for MMBIR is the presence of short regions of homology at the breakpoint. Although the exact sequence of the breakpoint on the short arm of chromosome 22 is not known, however, it is known that this portion of the genome is rich in tandem repeats with a shared micro-homology with other genomic regions that might be the substrate for MMBIR. We are aware that what we are proposing is a hypothesis that only the sequencing of the breakpoints and the identification of regions of micro-homology can confirm or disprove.

\section{Conclusions}

We have reported what we believe is a new case of pure duplication of $22 \mathrm{q} 13.2$, where we demonstrate that the combined use of FISH with both the copy number state and the genotype information, provided by SNP array, are not only important for the characterisation of the rearrangement and its origin, but also to shed light on the molecular mechanisms underlying cryptic chromosomal rearrangements.

\section{Consent}

Fully informed parental consent was obtained for the publication of this case report together with Fig. 1. A copy of the written consent is available for review by the editor of this journal.

\section{Abbreviations}

BAC: Bacterial artificial chromosome; BIR: Break-induced replication; DADAPI: Distamycin A and 4',6-diamidino-2-phenylindole; DAPI: 4',6-Diamidino2-Phenylindole, Dilactate; DSBs: Double strand breaks; FISH: Fluorescence in situ hybridisation; MMBIR: Micro-homologous-mediated BIR; NAHR: Nonallelic homologous recombination; NHEJ: Non-homologous end join; NOR: Nucleolus organiser region; SNP: Single nucleotide polymorphism; WISCH: Wechsler intelligence scale for children.

\section{Competing interests}

The authors declare that they have no competing interests.

\section{Authors' contributions}

CM carried out the bioinformatics analyses of SNP array data, researched the literature and wrote the manuscript. MT carried out SNP-array experiments. VB and GS performed cytogenetic and FISH analyses. EM and AP identified the patients and carried out the clinical characterization. EM also researched the literature and performed the clinical comparison with previously reported cases. GP interpreted FISH data, wrote the manuscript, designed and coordinated the study. All authors approved the manuscript.

\section{Acknowledgements}

We would like to thank the patient and her parents for their consent in participating in the study. We would like to dedicate this paper to the late Prof. Sergio Barlati, who contributed to the initial stages of this work. This work was supported by the Cytogenetic and Molecular Genetics Laboratory (LCGM) of Brescia University. 


\section{Author details}

${ }^{1}$ Biology and Genetics Division, Department of Molecular and Translational Medicine, University of Brescia, Viale Europa 11, 25123 Brescia, Italy. ${ }^{2}$ Pediatric Division, Spedali Civili, Brescia, Piazzale Spedali Civili 1, 25123 Brescia, Italy.

Received: 8 October 2014 Accepted: 26 June 2015

Published online: 07 July 2015

\section{References}

1. Gosden JR, Lawrie SS, Gosden CM. Satellite DNA sequences in the human acrocentric chromosomes: information from translocations and heteromorphisms. Am J Hum Genet. 1981;33(2):243-51.

2. Greig GM, Willard HF. Beta satellite DNA: characterization and localization of two subfamilies from the distal and proximal short arms of the human acrocentric chromosomes. Genomics. 1992:12(3):573-80.

3. Jones RS, Potter SS. Characterization of cloned human alphoid satellite with an unusual monomeric construction: evidence for enrichment in HeLa small polydisperse circular DNA. Nucleic Acids Res. 1985;13(3):1027-42.

4. Ravnan JB, Tepperberg JH, Papenhausen P, Lamb AN, Hedrick J, Eash D, et al. Subtelomere FISH analysis of 11688 cases: an evaluation of the frequency and pattern of subtelomere rearrangements in individuals with developmental disabilities. J Med Genet. 2006:43(6):478-89.

5. Abeliovich D, Maor E, Bashan N, Carmi R. Duplication of distal 22q. Am J Med Genet. 1989;32(3):346-9.

6. Barajas-Barajas LO, Valdez LL, Gonzalez JR, Garcia-Garcia C, Rivera H, Ramirez L. Sensorineural deafness in two infants: a novel feature in the 22q distal duplication syndrome. Cardinal signs in trisomies 22 subtypes. Genet Couns. 2004;15(2):167-73.

7. Biesecker LG, Rosenberg M, Dziadzio L, Ledbetter DH, Ning Y, Sarneso C, et al. Detection of a subtle rearrangement of chromosome 22 using molecular techniques. Am J Med Genet. 1995;58(4):389-94.

8. Cantu JM, Hernandez A, Vaca G, Plascencia L, Martinez-Basalo C, Ibarra B, et al. Trisomy 22q12 leads to qter: "aneusomie de recombinaison" of a pericentric inversion. Ann Genet. 1981;24(1):37-40.

9. Feenstra I, Koolen DA, Van der Pas J, Hamel BC, Mieloo H, Smeets DF, et al. Cryptic duplication of the distal segment of $22 q$ due to a translocation (21:22): three case reports and a review of the literature. Eur J Med Genet. 2006:49(5):384-95.

10. Fryns JP, De Backer D, Lemli L, Pedersen JC, Van den Berghe H. Partial duplication of the long arm of chromosome 22 (22q 13) with complete 22 trisomy phenotype. Acta Paediatr Belg. 1980;33(2):125-7.

11. Jensen PKA. A 5p;22q reciprocal translocation with a high risk for segregation of unbalanced offspring. Clin Genet. 1984;26(3):2.

12. Johnson MP, Greb A, Goyert G, Drugan A, Qureshi F, Sacks AJ, et al. Midtrimester diagnosis and anomalies in the dup(22q) syndrome: correlation of aneuploidy with low maternal serum alpha-fetoprotein and oligohydramnios. Am J Med Genet. 1990;36(1):94-6.

13. Okamoto N, Kubota T, Nakamura Y, Murakami R, Nishikubo T, Tanaka I, et al. 22q13 Microduplication in two patients with common clinical manifestations: a recognizable syndrome? Am J Med Genet A 2007;143A(23):2804-9.

14. Peeters $\mathrm{H}$, Vermeesch J, Fryns JP. A cryptic duplication 22 q13.31 to qter leads to a distinct phenotype with mental retardation, microcephaly and mild facial dysmorphism. Genet Couns. 2008;19(4):365-71.

15. Petek E, Kostl G, Mutz I, Wagner K, Kroisel PM. Characterization of a de novo partial trisomy 22q13-qter in a patient by microFISH. Clin Dysmorphol. 2000;9(1):55-7.

16. Rivera H, Garcia-Esquivel L, Romo MG, Perez-Garcia G, Martinez y Martinez R. The 22q distal trisomy syndrome in a recombinant child. Ann Genet. 1988;31(1):47-9.

17. Schinzel A, Niedrist D. Chromosome imbalances associated with epilepsy. Am J Med Genet. 2001;106(2):119-24.

18. Wieczorek D, Holtvogt J, Thonig S, Gillessen-Kaesbach G. A female patient with partial duplication 22 (q13->qter). Clin Dysmorphol. 1998;7(4):289-94.

19. Failla P, Romano C, Alberti A, Vasta A, Buono S, Castiglia L, et al. Schizophrenia in a patient with subtelomeric duplication of chromosome 22q. Clin Genet. 2007;71(6):599-601.

20. Rooney DE. Human cytogenetics a practical approach, vol. 2. New York: Oxford University Press; 1992

21. ISCN. ISCN 2013: An International System for Human Cytogenetic Nomenclature (2013). Basel: S Karger AG; 2013
22. Piovani G, Borsani G, Bertini V, Kalscheuer VM, Viertel P, Bellotti D, et al. Unexpected identification of two interstitial deletions in a patient with a pericentric inversion of a chromosome 4 and an abnormal phenotype. Eur J Med Genet. 2006:49(3):215-23.

23. Petryszak R, Burdett T, Fiorelli B, Fonseca NA, Gonzalez-Porta M, Hastings E, et al. Expression Atlas update-a database of gene and transcript expression from microarray- and sequencing-based functional genomics experiments. Nucleic Acids Res. 2014;42(Database issue):D926-32

24. Han K, Holder Jr JL, Schaaf CP, Lu H, Chen H, Kang H, et al. SHANK3 overexpression causes manic-like behaviour with unique pharmacogenetic properties. Nature. 2013;503(7474):72-7.

25. Mirza G, Imaizumi K, Ragoussis J. Partial trisomy 22 in a liveborn resulting from a rearrangement between chromosomes 6 and 22. J Med Genet. 2000;37(9):E22

26. Prasher VP, Roberts E, Norman A, Butler AC, Krishnan VH, McMullan DJ. Partial trisomy 22 (q11.2-q13.1) as a result of duplication and pericentric inversion. J Med Genet. 1995:32(4):306-8.

27. Bosco G, Haber JE. Chromosome break-induced DNA replication leads to nonreciprocal translocations and telomere capture. Genetics. 1998;150(3):1037-47.

28. Haber JE. Transpositions and translocations induced by site-specific doublestrand breaks in budding yeast. DNA Repair (Amst). 2006:5(9-10):998-1009.

29. Myung K, Chen C, Kolodner RD. Multiple pathways cooperate in the suppression of genome instability in Saccharomyces cerevisiae. Nature. 2001;411(6841):1073-6

30. Hastings PJ, Ira G, Lupski JR. A microhomology-mediated break-induced replication model for the origin of human copy number variation. PLoS Genet. 2009:5(1):e1000327.

\section{Submit your next manuscript to BioMed Central and take full advantage of:}

- Convenient online submission

- Thorough peer review

- No space constraints or color figure charges

- Immediate publication on acceptance

- Inclusion in PubMed, CAS, Scopus and Google Scholar

- Research which is freely available for redistribution 